\title{
Research on Virtual Three-Dimensional Clothing Styles and Fold Effect
}

\section{Show}

\author{
Jingjing $\mathrm{Li}^{1}$
}

${ }^{1}$ Apparel Management Department of Jiangxi Clothing Institute, Nan Chang, Jiang Xi, 330201

KEYWORDS: Virtual, Three-Dimensional Clothing, Style Design, Fold effect, CAD

\begin{abstract}
Since the birth of garment computer aided design, production efficiency and product quality garment processing has been greatly improved and faster. Its principle is the human body consists of a simulation model, the use of a switching supply tool costume design environment. Such fashion design is no longer a simple paper model 2D, but in a human body can be worn on the model's clothes displayed, providing unprecedented functional system for the apparel development, improve the quality of apparel products and efficient Productivity. In conclusion, what we will build a three-dimensional surface model style, and the use of light materials and processing methods to achieve true three-dimensional style graphics effects; plus a variety of other means to achieve the modification of three-dimensional models, to meet the individual needs of fashion design; to show the clothing style fold effect, make clothing to artistic ability has been greatly improved, but also to achieve a more theoretical basis and high practical value contemporary textile engineering computer-aided design.
\end{abstract}

\section{Introduction}

Our study shows for the three-dimensional virtual clothing styles and fold effect is provided by the computer graphics and dynamic model of intelligent computing in the textile field a theoretical basis for the application of such clothing and fabric design has a more profound theoretical significance. In the design, production, marketing and other areas of textile and apparel prospect of having a large range of fabrics and garments in product design, development, marketing and other areas have greatly improved efficiency, this efficiency allows extensive customization of personalized clothing can be achieved and became one of the commercial development prospects of textile and clothing.

\section{The Research Status of Virtual Three-Dimensional Clothing Style Folds}

Generally information technology as a three-dimensional virtual clothing and garment engineering technology cross-cutting areas, it refers to the use of virtual reality and simulation technology, graphics technology and other methods of clothing and fabrics simulation to achieve a costume design and technology of the type of display . In the early years of the 20th century, physical modeling and geometric modeling appeared in the clothing simulation Circle. According to physical simulation and improve the interaction between fabric mechanical properties themselves and with the external environment such as air resistance, gravity and so on. This is the major reason for the physical simulation method can better simulate the static and dynamic clothing, especially clothing dress dynamic effect, so widely used in animation, games and clothing display. 
Of course, from a technical perspective, geometric modeling method are three ways to use creativity to generate three-dimensional clothing, clothing and three-dimensional scanning is based on scanning of the existing clothing and use their technology to get the surface reconstruction, belongs one reverse engineering category, and there is no obvious creative design concepts into the inside. So from the point of view of clothing dress effect, geometric modeling methods are generally only show a static effect.

Virtual three-dimensional garment can be divided into the following three aspects: one is in the field of computer animation and entertainment, computer-generated actors because the need for rapid and convenient tool to dress, dress up, make synthetic actors look closer to the real world character; the second aspect is, in the CAD field, modified forms of textile simulation technology has more stringent requirements, such as three-dimensional garment $\mathrm{CAD}$ and digital fashion customize these; for e-shopping platform, users can through online virtual costumes, interactive way to a free replacement of their favorite costumes and try in the process, they can also express their views and express their own modifications of clothing, so as to achieve a more perfect dress phenomenon; that is, a third aspect of things applications, virtual dressing system according to the customer's personal preferences and purchase history usually recommend customers similar style of dress clothes, and even customers can also design their own clothing styles, so not only can save a lot of marketing and advertising costs, but also e-commerce operation of enterprises have a good role in promoting.

\section{The Research Content of Virtual Three-Dimensional Clothing Style Folds}

For now, the commonly used CAD garment design process is designed to simulate the traditional costume of the route. Our first application is a two-dimensional design software to map out clothing styles of dress style renderings or simple planar configuration diagram, then the idea of designer clothing image visualization out a preliminary work to complete the design concept; again by experience abundant through playing board software designer to draw two-dimensional pieces; and finally the input module fitting three-dimensional, virtual display dress stitching effect, this process is equivalent to the production of a series of model clothes. It notes that the transition from flat styles to chart conversion is a two-dimensional pieces of cutting the need for professional knowledge, because this is an artificial conversion process. And displaying three-dimensional effect is fashion design three-dimensional basis is formed in which the fibers collection plate was made with certain dimensions of the development of a fabric body fabric modified forms of simulation technology. Many kinds of fabric, because it is formed of material, shape, color, changing the structure of these methods, but it is the basic material is composed of clothing. Second calculated by two-dimensional kind of clothing designed and necessary conditions have been calculated on the basis of the clothing sets three-dimensional models of three-dimensional data display style 3D. Of course, two-dimensional data into three-dimensional data and this is a process of information transfer clothing styles, clothing after getting three basic data form a "virtual capsid," wants a complete clothing style, you need a two-dimensional models plan to modify the outline style, in order to achieve three-dimensional models of data points.

Fold is a general feature of the appearance of clothing, the clothing can well reflect the authenticity of clothing, can also express the designer's creative intention to getting more clothing based on 3D mesh clothing folds simulation technology get more practical solution. In the simulation of clothing fold effect, divided into two categories, static simulation effect is one of a kind, it is widely used in some large apparel companies holding function, add the product upload or skirt, making it easy to observe the effect in real time, but it the form does not change with time will 
change. Another one is that simulation of dynamic effects, but such simulation allows simulation of clothing with the passage of time in the outside world have a role or a flowing drape effect, one important effect is the implementation of collision detection between the garment and the virtual human. For now, this state is still relatively frontier attributable to clothing dynamic effects simulation technology in one. The folds in the clothing can also be produced by the process of natural wear, due to changes in the bending deformation of the flexible fabric and human limb movements arising. For example, in the abdomen, waist and knees we have a lot of downloading of such folds presence of necessity. Of course folds may also be artificial shirring, playing Tong, transports and collects, processes overfeed these folds formed by the method, such man-made garment folds also constitute one element, is a change in the fabric closer to real life . And usually they have some kind of decorative folds, is the embodiment of beauty, is widely used in the many national costumes. Not only are different types of folds produced, wrinkles will be different because the material, position, shape, technology and other techniques, which produce different aesthetic, but also have different effects on the clothing style. In various historical periods, different cultures of East and West, so that wrinkles have different expressions and the use of history and culture also have different interpretations. Especially in recent years, women's design, the folds become one of the most popular style and a lot of fashion borrowed from the fold to express their design ideas, create their own style, folds designer apparently has become the most expression force fashion language.

Clothing styles of clothing design, also known as image design or costume design effect is usually performance clothing appearance the state is part of the surface of fashion design. This time the designer through the use of CAD software provides a variety of tools, more efficient completion point, line, polygon drawing and filling color costume design, costume changes and the overall picture retouching, and ultimately achieve the effect of a high quality mapping and information on the use and management of information.

\section{The Study Trends of Virtual Three-Dimensional Clothing Style Fold}

Throughout the use of computer-aided design and costume as the rapid development of society, the three-dimensional garment CAD is unstoppable torrent. Whether from the designer's needs as well as the requirements of customers, the three-dimensional computer-aided design clothing will be an inevitable trend, and this will be the beginning of a change of clothing design industry. Of course, for autonomous intelligent clothing research systems, in order to make clothing style stitching to play better, give customers more intense visual effects, costume design greatly enhance the efficiency of the implementation of the three-dimensional garment is the need to go way. Of course, the study of three-dimensional virtual clothing has a long history and I believe its future trends and directions of development more worthy researcher careful thought.

\section{Conclusion}

With the virtual three-dimensional garments to showcase the technology more in-depth research, intelligent three-dimensional virtual display is its clothing trends, designers have the freedom to design their own clothing apparel browse through virtualization technology showcase effect in three-dimensional space, and improve the efficiency of the value of quality clothing, which will have broad application prospects in individual design and customization, traditional clothing digital display and other areas of fashion clothes. And the study of three-dimensional virtual display clothing on our theoretical and practical significance has greatly improved. Clothing is also a 
three-dimensional cross-discipline, I believe that with the development of science, we work together to give full play to the characteristics of the various disciplines, through research, is bound to open up a three-dimensional display from the clothing of a new road.

\section{REFERENCE:}

[1] Zhang Tianrui. virtual clothing styles and three-dimensional fold effect display research [D]. Donghua University, 2007.

[2] Yang Tianhong. based on three-dimensional fashion style dress effect texture mapping study [D]. Donghua University, 2007.

[3] Wang Jin. Integrableware sketch interactive 3D garment design method and reuse technology research [D]. Zhejiang University, 2008.

[4] Wang Xiaoyang Zhu Guojin, Chen Xun. three-dimensional garment prototype garment liberal amount generated model [J]. Microcomputer Information, 2007,22: 202-203 + 197.

[5] Hu Jianwei, Chen Qiang. based on realistic virtual three-dimensional effect of the clothing simulation [J]. style design of Computer Engineering and Design, 2008, 23: 6142-6145.

[6] Han Xu, Hu Yong, Li Zuhua. three-dimensional garment CAD technology [J]. Guangxi Textile Science, 2008,02: 58-60. 